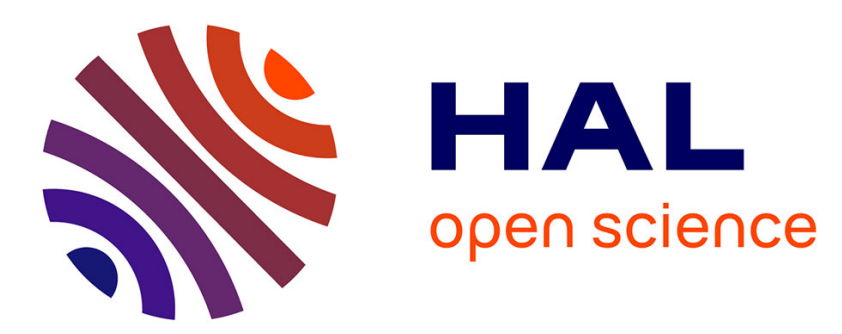

\title{
Evaluation of block interleaving techniques for robust image communication in wireless camera sensor networks
}

Eric Orellana-Romero, Javier Sanmartin-Hernandez, Cristian Duran-Faundez, Vincent Lecuire, Katherine Zapata-Quiñones

\section{To cite this version:}

Eric Orellana-Romero, Javier Sanmartin-Hernandez, Cristian Duran-Faundez, Vincent Lecuire, Katherine Zapata-Quiñones. Evaluation of block interleaving techniques for robust image communication in wireless camera sensor networks. IEEE Conference on Wireless Sensors, ICWiSE 2014, Oct 2014, Kuala Lumpur, Malaysia. hal-01093292

\section{HAL Id: hal-01093292 \\ https://hal.science/hal-01093292}

Submitted on 10 Dec 2014

HAL is a multi-disciplinary open access archive for the deposit and dissemination of scientific research documents, whether they are published or not. The documents may come from teaching and research institutions in France or abroad, or from public or private research centers.
L'archive ouverte pluridisciplinaire HAL, est destinée au dépôt et à la diffusion de documents scientifiques de niveau recherche, publiés ou non, émanant des établissements d'enseignement et de recherche français ou étrangers, des laboratoires publics ou privés. 


\title{
Evaluation of Block Interleaving Techniques for Robust Image Communication in Wireless Camera Sensor Networks
}

\author{
Eric Orellana-Romero*, Javier SanMartin-Hernandez*, Cristian Duran-Faundez ${ }^{\dagger}$, Vincent Lecuire ${ }^{\ddagger}$ and \\ Katherine Zapata-Quiñones ${ }^{\S}$ \\ *Escuela de Ingeniería Civil en Automatización, Universidad del Bío-Bío, Concepción, Chile \\ Email: \{eorellan, javsanma\}@alumnos.ubiobio.cl \\ ${ }^{\dagger}$ Departamento de Ingeniería Eléctrica y Electrónica, Universidad del Bío-Bío, Concepción, Chile \\ Email: crduran@ubiobio.cl \\ $\ddagger$ Centre de Recherche en Automatique de Nancy, Université de Lorraine, CNRS, Nancy, France \\ Email: vincent.lecuire@univ-lorraine.fr \\ $\S$ Magister en Ciencias de la Computación, Universidad del Bío-Bío, Chillán, Chile \\ Email: kzapata@ubiobio.cl
}

\begin{abstract}
We study block interleaving techniques for enhancing robustness against packet loss on wireless vision sensor networks. We evaluate three techniques: DSJ-AL interleaving, Turner and Peterson (TP) technique, and Torus Automorphisms (TA). Results in terms of image quality, obtained trough extensive simulations over monochromatic images, show the improvements provided by interleaving techniques in comparison with a sequential communication, with a slight favor to TA.
\end{abstract}

Image communication; block interleaving; error resilience; wireless camera sensors; packet loss

\section{INTRODUCTION}

Wireless Sensor Networks (WSNs) consist of a large number of small electronic devices with sensing, communication and processing capabilities [1]. Usually, their application fields relate to simple scalar measures such as temperature, pressure or humidity [2], however, advances in microelectronics allow low cost capture and transmission of digital images, making possible Wireless Vision Sensor Networks (WVSNs) [3]. Such networks introduce a large number of open challenges, because the many problems present in traditional WSNs are increased by the use of more complex sensors (cameras) and the need to deal with the thousands of bytes composing digital images, with similar resource constraints.

From the many issues concerning WSNs, the problem of robustness against packet loss requires special attention. Indeed, in [4], authors argue that packet losses are inherent in WSNs. Moreover, some real-world environments report loss rates surpassing the $70 \%$ of the transmitted packets [5], thus error resilience is a critical issue.

Packet loss can occur due to many factors including channel errors, collisions and congestion. In WVSNs, these events, together with the vast amount of generated data (pixels), present problems to the communication process. Indeed, while to codify and communicate a single scalar measurement (such as temperature or presure) 2 or 3 bytes are largely enough, images are codified in thousand of bytes, grouped in hundreds of packets, which in case of getting lost adversely affect the quality of received images at the decoder side (more important, when image compression is applied), and their retransmission is power expensive.

Due to these statements, image transfer on WSNs requires communication techniques that maintain the quality of the obtained images, taking into account the problem of packet loss and traditional constraints of this kind of network. In some very constrained systems, traditional implementation of ACK or FEC-based methods is unsuitable because they require additional energy and time consumptions. Thus, it is important to evaluate alternative methods and adaptations of traditional techniques allowing more efficiently to deal with this issue.

A low-cost and effective solution for enhancing robustness of an image communication system is block interleaving. A block interleaving technique disrupts the traditional sequence of packetization-communication of an image blocks. It is based on the inherent spatial information redundancy of digital images. As neighboring blocks of pixels are sent in different data packets, there is a better probability of receiving neighboring blocks of a lost one, thus to count with enough information to estimate its value. In the literature, many works have adopted interleaving methods to increase robustness of a communication system but non of them compares these methods in a WVSNs context. Also, interleaving effectiveness seems to be assumed, and the evaluation of the robustness provided by the interleaving method is usually not rigorous. This paper addresses the problem of robustness in WVSNs evaluating various image interleaving methods found at the literature, addressing theoretical issues, and comparing them through extensive simulation, in terms of quality of the received images, with different testing parameters. The rest of this paper is organized as follows: Section II presents related works to the adoption and evaluation of image interleaving for 
robust image communication on WVSNs. Section III gives theoretical background on block interleaving, describing the selected block interleaving techniques studied in this work. Further analysis and parameters of evaluation for each technique are presented in Section IV. Simulation results are shown in Section V, presenting results for each method in terms of image quality for best parameter combinations found and results distributions associated to different result ranges. Finally, Section VI concludes this work.

\section{RELATED WORKS}

Various techniques can be adopted to allow reliability in a communication system. ARQ-based schemes can be adopted in order to ensure the reception of the packets sent by a camera source to the sink, at each hop in a path but this could be excessively expensive in terms of energy consumptions and delay. Energy savings can be achieved if a semi-reliable strategy is adopted [6], but the number of retransmissions needed to ensure the arrival of high priority packets can lead to similar consequences of a full-reliable communication, if no other error resilient techniques is applied. Similar consequences may occur by the transmission of redundant packets. For example, in [7], the problem of error probability in image transport is addressed by generating diverse copies of the same packet and transmitting them through diverse nodes in a cluster-based network. Copies are sent by a cluster head to various nodes, then, the copies are transmitted to the next cluster head towards the sink. Of course, if packets are lost, other copies could be received by the next cluster head. Such a technique ensures the reception of a good quantity of information packets at the decoder side, but the transmission of redundant packets and the required protocol complexity affects resource consumptions.

Multiple properties of digital images could be taken in advantage in order to provide resistant transport to communication errors in accordance with WVSNs specifications. In general, robust mechanisms proposed in the literature are based in the fact that, to many WVSNs applications, what is relevant is to obtain useful versions of the original images instead of lossless information. For example, the image communication scheme proposed in [8] takes advantage of this correlation. It is based on SPIHT coding of independent data blocks generated from parent-child relationships of wavelet coefficients. Each codified block generates an independent data stream, thus channel errors affect only the involved block of coefficients, reducing the impact of data loss.

In various works, inherent spatial correlation between blocks of pixels and their neighboring ones is exploited. In this paper, we address block interleaving as a technique for enhancing robustness of an image communication system. In the WVSNs context, work in [9] shows a chaotic interleaver based on a discretized Baker map. New coordinates for a block are calculated by an equation taking as parameters original indices and a secret key vector $\left[n_{1}, \ldots, n_{k}\right]$ with $n_{1}+\cdots+n_{k}=N, N$ being the number of blocks in an image row). This related work is interesting because it is specially conceived for Zigbee-based WVSNs, however,

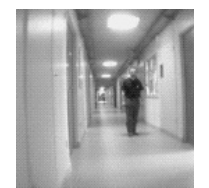

(a) Original image

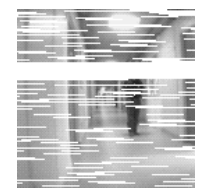

(b) Received bitmap

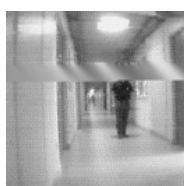

(c) Reconstructed version
Fig. 1. Example of the effect of packet loss on sequential image transmission.

the enormous quantity of possible parameters combinations (secret key) makes difficult its exhaustive evaluation, thus this technique was not considered in the scope of this paper. Future studies should consider this technique.

In [5], a chaotic Torus Automorphisms is adopted to improve robustness against packet loss in a Cyclops/Mica2 network. Results show that improvements in the quality of the reconstructed images can be achieved in presence of high packet loss rates, without excessively increase resource consumptions. Same technique is applied in [10] for the case of images compressed in independent blocks of pixels. In this work we will evaluate three representative interleaving technique found in the literature.

\section{BLOCK INTERLEAVING TECHNIQUES}

Let us illustrate the effect of packet loss in the transmission of a digital image with the example in Figure 1.

The captured image of Figure 1a is received with losses, obtaining the bitmap of Figure 1b. After applying an error concealment method (as those mentioned by [11]) it is possible to obtain the reconstructed version of Figure 1c. Notably, the quality of the received image is greatly degraded. In cases where lost data packets are isolated a good reconstruction can be achieved (see, e.g., the half-bottom of the reconstructed image), but in places where big areas are lost (in some cases due to burst losses), reconstruction is impossible without appropriate codification at the source side. Block interleaving can be applied to mitigate packet loss effects. The principle of block interleaving is to transfer adjacent blocks of pixels in the original image (codified or not) into data packets as far as possible. The ideal situation is not finding two neighboring blocks from the original image in the same packet, so there is better probability of receiving neighboring blocks to a lost one (and thus, of better estimate the missing values). Virtually, an interleaving algorithm performs this task disrupting the bitmap of the original image, assigning different positions to adjacent pixels, and thus increasing the probability of transmitting in different packages, as illustrated in Figure 2.

For a similar scenario of the illustrated in Figure 1, the image in Figure $2 \mathrm{a}$ is sent through a non-reliable channel, but this time being interleaved first. The interleaved version of the original image is shown in Figure $2 \mathrm{~b}$. At the decoder side, interleaved bitmap suffers same packet loss of previous example, as shown in Figure 2c. After performing inverse interleaving (reallocation of blocks of pixels to the original position), we obtain the bitmap of Figure $2 \mathrm{~d}$. In this new map, missing information is noticeably better isolated, giving 


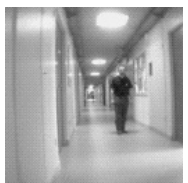

(a) Original image

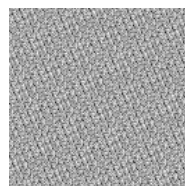

(b) Interleaved image

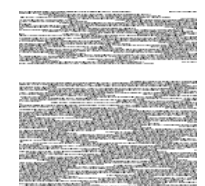

(c) Received bitmap

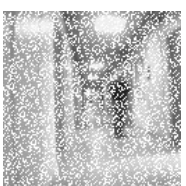

received bitmap
Deinterleaved

(e) Reconstructed version

Fig. 2. Example of the application of image interleaving in an image communication scenario in presence of high packet loss.

as a result (after the error concealment application), the approximate version of Figure 2e. This example illustrates the improvement allowed by a block interleaving in comparison with the sequential transmission case.

Of course, the obtained results will depend on the adopted interleaving technique. In the following, we explain and evaluate three interleaving methods applied in the literature for robust image communication.

\section{A. Turner and Peterson (TP) interleaving}

The encoding algorithm introduced by Turner and Peterson (TP) [11] consists into modify the original spatial positions of each pixel by two parameters. The first, byteoffset, specifies the distance between adjacent pixels (in the original image) into a single packet, i.e., it specifies the number of pixels not included between two pixels added to a packet. The second parameter, packetoffset, indicates the distance (in pixels) between the first elements of two adjacent packets, starting the generation of a new one packetoffset pixels after the first element of the previous packet, diminishing the effects of burst packet loss. In the paper, this method is not applied to WSNs, but given its simplicity, its implementation is perfectly possible to this kind of network. Then, for $I(H \times W)$ images, $I=\left\{p_{0}, p_{1}, \ldots, p_{H * W-1}\right\}$, where $p$ corresponds to the pixels of $I$; the $i$-th packet $P_{i}$, such that $i \geq 0$, contains $m$ elements, being $P_{i}=\left\{p_{j}, \ldots, p_{j+2 * \text { Byteoffset }}, \ldots, p_{j+(m-1) * \text { Byteoffset }}\right\}$, with $j=i *$ Packetoffset.

\section{B. DSJ-AL interleaving method}

As a part of the Dual $\underline{\text { Stream }} \underline{\mathrm{J} P E G} \underline{\text { Adaptation }} \underline{\text { Layer, DSJ- }}$ AL, method, Posnak et al. [12] incorporate an interleaving method in which, for an image of $H \times W$ blocks, two originally adjacent blocks are separated by a parameter step. This is performed as follows: Given a packetized block in coordinate $\left(x_{i}, y_{i}\right)$ (it is assumed that the first position is $(0,0))$, the coordinate of the following block to be packetized, $\left(x_{i+1}, y_{i+1}\right)$, is calculated by using Equations 1 and 2 .

$$
\begin{aligned}
y_{i+1} & =\left(y_{i}+\text { step }\right) \bmod W \\
x_{i+1} & =x_{i}+\left(\frac{y_{i}+\text { step }}{W}\right)
\end{aligned}
$$

Originally, this technique is applied to interleave codified blocks of pixels, but it can also be used to interleave individual non-codified blocks. For proper generation of new positions, it is indicated that, once the maximum number of rows $(H)$ for $x_{i+1}$ the next pixel to be assigned will be the following to the first on the current cycle. For example, assuming that the first coordinate in the current cycle is $(0,0)$, once $x_{i+1}>H$ exceeds this amount, the coordinate generated for the next pixel should be $(0,1)$.

\section{Torus Automorphims (TA) interleaving}

Torus Automorphims (TA) are highly chaotic systems, that can be used as permutation functions in order to implement block interleaving. In this work, we evaluate Arnold's Cat map-based automorphisms applied in [5]. Given an image $I$ of $N \times N$ blocks, and $\{x, y \in \mathbb{N} / 0 \leq x<N \wedge 0 \leq y<N\}$, each pixel with coordinates $(x, y)$ is stored in a new position $\left(x^{\prime}, y^{\prime}\right)$ calculated by Equation 3 .

$$
\left(\begin{array}{l}
x^{\prime} \\
y^{\prime}
\end{array}\right)=\left(\begin{array}{cc}
1 & 1 \\
k & k+1
\end{array}\right)^{n}\left(\begin{array}{l}
x \\
y
\end{array}\right) \bmod N
$$

\section{FINDING EVALUATION PARAMETERS}

An important topic in this work is to evaluate the performance of the selected interleaving methods. In image communication through WVSNs these techniques are used as a tactic to obtain robustness against losses, thus their performance is directly related to the quality of the resulting images at the receiver side. Specifically, the stated problem consists on the choice of one (or various) interleaving techniques and its parameters, so that the quality of the obtained images is within acceptable ranges. Due to the above, we study the behavior of the described techniques with multiple images and loss patterns, using the simulation framework Sim-LIT [13]. To assess conditions close to reality, 122 loss patterns obtained from real-world experiments (see full description in [5]) and monochromatic (8bpp) $128 \times 128$ versions of the 44 test images found at [14] are used. The following subsections describe the adopted range of parameters used in our simulations.

\section{A. TP method parameters}

In TP, there is a large number of parameter combinations available for selection, making difficult the choice of good combinations. Moreover, when testing TP with arbitrary parameters, for some particular parameter combinations, coordinates generated by the algorithm are equal for various different pixels, overwriting the first ones.

In order to obtain a list of valid parameter combinations, we observe the reallocation of pixels in a $128 \times 128$ boolean matrix, initiated to False. Then, generating coordinates with 
TP technique, True values are assigned to each new generated position, labeling the combination of parameters as invalid if any overwriting occurs. This study is performed for combinations of parameters included in the ranges $0 \leq$ Byteoffset $<16,500$ and $0 \leq$ Packetoffset $<$ 16500 , using packet size of 27 bytes. Finished this evaluation, the amount of correct pairs is greater than 8300 , being necessary to check the existence of identical interleaving patterns generated using different combinations of parameters. After eliminating equivalent combinations (parameters who through same interleaving patterns that other), valid pairs are decreased to 8192 . We must note that pairs qualified as valid parameters are bounded by a value of $128 \times 128$, equivalent to the length of adopted images. From the previous study, we obtained a table with valid pairs within the specified range.

\section{B. DSJ-AL interleaving parameters}

In order to evaluate the behavior of DSJ-AL interleaving, we use a $128 \times 128$ image. Simulations varying its unique parameter step in the range $[1,17000]$ were performed, calculating the PSNR between the mixed and the original images. Infinity results (cases where resulting and original images are equal) are obtained for step $=1$ and step $\geq 128 \times 128$. Comparing the results with DSJ-AL behavior, we note coherence between them, because, for step $=1$ there is no offset in the pixels, and for step $\geq 128 \times 128$ a greater distance between pixels to the total length of the image, is generated. This behavior generates unmixed images for ste $p=1$ and for step $\geq(N \times N)$ images. In this way, the evaluation range is defined to $1<$ step $<(N \times N), N \in \mathbb{N}$, for a $N \times N$ image.

\section{TA interleaving parameters}

Because of the presence of two parameters ( $k$ and $n$ ), there is a great amount of combinations, making difficult to determine a parameter pair which generates good results. One aspect to consider is that the technique is based on toroids. This suggests that the results are generated in cycles, allowing narrow each parameter to a range. Generally speaking, it is expected that there is a maximum value $k$ with its corresponding $n$ value, that limits the results, reducing the amount of possible choices. Thus, the method used to define limits is based on Equation 3, where it is possible to note that the interleaved positions $\left(x^{\prime}, y^{\prime}\right)$ are determined by the matrix $\left(\begin{array}{cc}1 & 1 \\ k & k+1\end{array}\right)^{n}$ $\bmod N$. Indeed, when this matrix corresponds to the identity, it causes $x^{\prime}=x$ and $y^{\prime}=y$, generating the same original image after interleaving. To determine the parameter values that enforce this condition, Equation 3 is evaluated for an arbitrary range of values (not considering $n \neq 0$ ), storing the first parameter $n=T$ that generates the identity matrix, for each value of $k$ in a bounded parameter list. This approach is carried out for images of size $128 \times 128$ pixels, evaluating the parameters for the range $0 \leq k \leq 300 \mathrm{y} 1 \leq n \leq 300$.

When analyzing the generated data, a pattern for $T$ values when varying $k$ is determined defined by $T(k)=T(k+R \times$ $128)$, with $R \in \mathbb{N}$ y $0 \leq k \leq 128$. That is, the sequence of values of $n$ that generate an original image for different values of $k$ is repeated each 128 increments from this last one. Given this, it is possible to establish as limit for $k$ the value 128 in images of $128 \times 128$ pixels. For the parameter $n$, it is detected that the values for which we obtain the same original images $n=T$ are discrete $(3,4,6,12,8,16,24,32,48,64,96$, 128 ) and they have as superior limit 128 , so that, for each value of $k$, in images of $128 \times 128$ pixels, an original bitmap is obtained, at least once, when $n=128$.

After performing similar experiments with $N=32, N=64$ and $N=256$, it is possible to affirm that for square images of $N \times N$ blocks, with $N=2^{x} / x \in \mathbb{N}$, the range of $k$ values producing different interleaved patterns can be limited to $0 \leq k \leq N$. Moreover, it is observed that the discretized nature of $T$ is maintained, adding, or eliminating, values to the set of frequencies, for a given $N$. In this way, for $N=32$, resulting $T$ values are in the set $F_{32}=$ $\{3,4,6,8,12,16,24,32\}$, for $N=64, F_{64}=F_{32} \bigcup\{48,64\}$, for $N=128, F_{128}=F_{64} \bigcup\{96,128\}$ and for $N=256$ they are given by the set $F_{256}=F_{128} \bigcup\{192,256\}$. Generalizing, to image interleavings with $N=2^{x} / x \in \mathbb{N}$, the set of frequencies for value $T$ are limited by $T=N$ and corresponds to the set $F_{N}=F_{N=2^{x-1}} \bigcup\left\{2^{x-1}+2^{x-2}, N\right\} / x>2$.

\section{Simulation Results}

Once the range of different parameter combinations for evaluation is defined, we proceed to assess which configuration(s) allows generate better results when recovering a transmitted image, so we can count on the needed information to obtain the required results. For this, the following procedure is performed for each method, evaluating obtained results looking for the best parameters in each case.

\section{A. Generic evaluation process}

Using the described set of 44 images and 122 loss patterns, we simulate with Sim-LIT the transfer of each of the images for each of the available loss patterns, using for that interleaving methods with parameters combinations as described in Section IV (for $H=W=N=128$ ), depending on the employed method. Average value of the correctly received neighboring pixels to a lost one was adopted as error concealment technique. between the resulting and the original images are obtained, for each simulation, assessing image quality for the different packet loss patterns and for the different configurations of the interleaving methods. We define a packet size of 27 bytes which corresponds to the default payload value of the Cyclops camera firmware. Once obtained these data, parameters providing the best results are evaluated. To this, the mean PSNR of the 44 reconstructed images is calculated for each simulation. In this way, a set of PSNR measures is obtained to measure the performance of each parameter combination under different channel conditions (loss patterns). Once the set of values that represents the different valid combinations of parameters is generated, it is necessary to compare their performance to determine a combination who gives the best results. To this, we adopted 


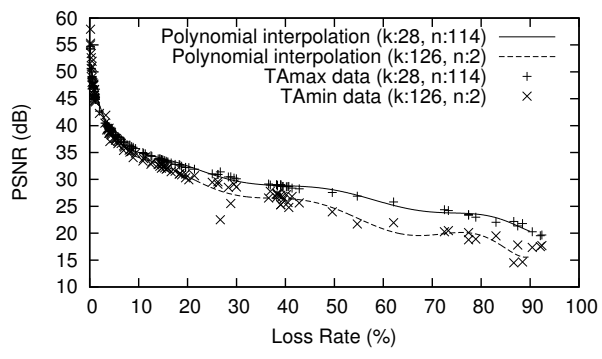

Fig. 3. Results of the best and worst pair of parameters, using TA interleaving.

GNU Octave software, generating a curve representing the obtained points after simulation, adopting to that polynomial functions of degree 8 , with function polyfit(). Thus, to determine the best pair of parameters the area under each curve is calculated, using functions polyout () to construct the polynomial function (using calculated coefficients with polyfit()), and quad() to calculate the defined integral in the range 1 to 90 (loss rates), determining the biggest area under each curve, and thus, the parameters generating the best results. We must note that this evaluation technique is not valid in every case, because it adopts an approximate representation method. However, the obtained results from this analysis are valid for most of the studied cases. To analyze the data produced after simulation in a generic manner, interpolated curves for each valid parameter are generated and the area under each of these is calculated. After that, the range of obtained areas is divided in 30 intervals and histograms are constructed with these data, showing the number of parameters for which areas are obtained within each group.

\section{B. Parameter evaluation of Torus Automorphims}

After performing the described procedure, the parameters providing a better value when calculating the integral of the interpolated curves are $k=28$ and $n=114$, obtaining an area under the curve of 2681 . In the same way, parameters $k=126$ and $n=2$ generate the minimum area value being of 2194. In the graphics of Figure 3, simulation data, along with interleaved curves for the best and worst parameters combinations is shown.

\section{Parameter evaluation for DSJ-AL interleaving}

DSJ-AL interleaving has a unique parameter, $s$. Following the procedure of Section V-A, it is obtained $s=92$ as the best parameter, reaching a maximal area under the interpolated curve equal to 2657. Moreover, the minimal area is determined, reached by the interpolated curve calculated with parameter $s=15082$, with a value of 2116. Graphics of Figure 4 shows simulation data, along with interleaved curves for the best and worst parameters combinations.

\section{Parameter evaluation for TP method}

TP method counts on two parameters, ByteOffset y Packet $O f f$ set. After applying the evaluation procedure, the pair giving the best results, measured through the calculation

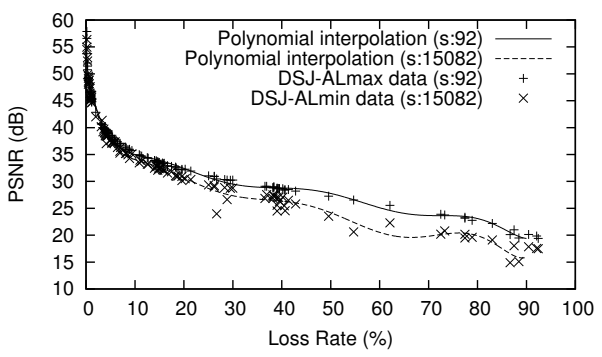

Fig. 4. Results of the best and worst pair of parameters, using DSJ-AL interleaving.

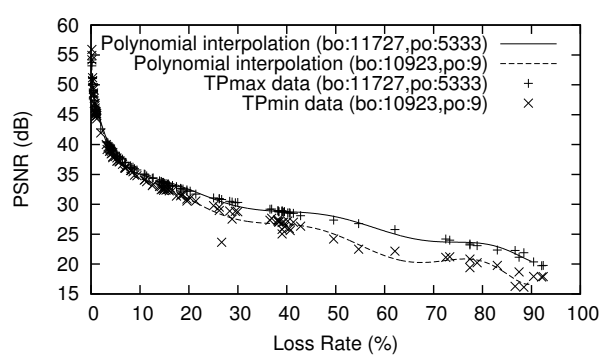

Fig. 5. Results of the best and worst pair of parameters, using TP interleaving.

of the interpolated curve's integral, is ByteOff set $=11727$ and PacketOffset $=5333$, obtaining an area under the curve of 2683 . On the other way, parameters generating the curve representing worst qualities are ByteOf f set $=10923$ and PacketOff set $=9$, reaching a respective area of 2216 . Figure 5 shows the respective results.

\section{E. Results comparison}

To compare the evaluated interleaving methods and a sequential image transmission (without interleaving), Figure 6 shows all obtained data using the best parameters of each method and the data generated when a sequential (noninterleaved) communication is applied. From this, it is possible to say that the all evaluated interleaving techniques generate better average results than the sequential transmission, when packet loss rates surpass $15 \%$. Below this value, all transmission strategies are quite similar. Regarding interleaving techniques, it is clear that generate similar results, however, there are differences over $40 \%$ of packet loss. Thus, for loss rates in the range between $40 \%$ and $80 \%$ TA generates slightly better results than their peers, however, TP method throw quite near results. Meanwhile, DSJ-AL is the one with a lower performance compared to other interleaving methods. Table I summarizes the maximum, minimum, and mean of the areas under the interpolated curves.

Notably, these are the maximum for each of the technical values, so to generate a conclusion on the best performance is necessary to evaluate the above results as a whole. Histograms of Figure 7 show the different distributions of the quantities of parameters generating areas under the interpolated curves for the different defined intervals. From the results, we observe that the methods having a bigger quantity of parameters giving 


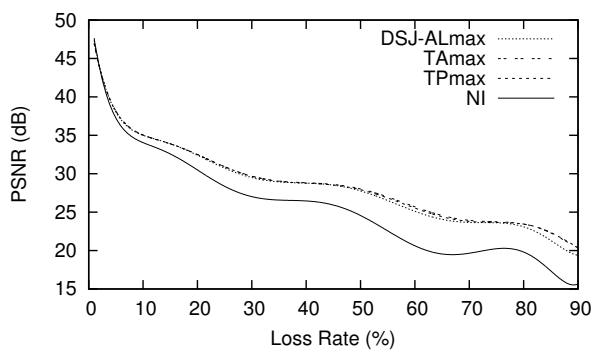

Fig. 6. Results for sequential and interleaved image transmission using best parameters.

TABLE I

SUMMARY OF THE RESULTING AREAS UNDER THE CURVE FOR EACH EVALUATED TECHNIQUE.

\begin{tabular}{|c||c|c|c|}
\hline Technique & Min. & Max. & Mean \\
\hline TA & 2194 & 2681 & 2531.69 \\
\hline DSJ-AL & 2116 & 2657 & 2323.26 \\
\hline TP & 2216 & 2683 & 2532.42 \\
\hline
\end{tabular}

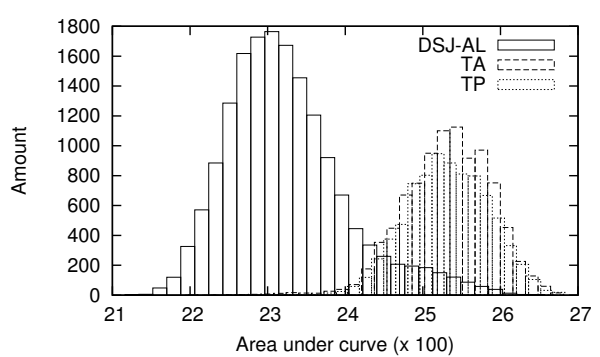

Fig. 7. Histogram of areas under the curve for each method.

results near the maximum are TA and TP. In the case of DSJ-AL, the major quantity of results are near the minimum. Comparing the best evaluated methods, we can say that TA stands out above TP, because its distribution throws a larger quantity of parameters giving areas near the maximum.

\section{CONCLUSION}

The conclusions generated are defined based on image transfer simulations for different cases, considering the quality of the final image as evaluation metric, measured by PSNR. Analyzing the simulated results for each technique, their methods of operation, and better operating range parameters are determined in terms of the quality of the final image in the receiving node. As a result, we observe that all these methods give better results than the sequential transmission for a loss rate greater than $15 \%$. Below this value the results of the final image qualities are very similar for all the evaluated cases. Regarding the difference between the interleaving algorithms, it is determined that gaps are generated in the final quality of the images transmitted with a packet loss superior to $40 \%$. For the evaluation of TA is determined that the parameters that generate better quality in the final image correspond to $k=28$ and $n=114$, while for evaluation Turner and Peterson interleaving is determined that the parameters that generate better quality in the final image are byteOff set $=11727$ and packet $O f$ feset $=5333$, considering as indicator the integral of the curve interpolated by a polynomial of degree 8 for each of the simulated data. For the evaluation of DSJ-AL interleaving results show that the best performing parameter in terms of the quality of the final image is $s=92$, based on the same study generated for the above methods. Generally, after comparing the number of parameter combinations that generate near maximum delivered by each best result for the methods studied and compare the interpolated curves generated for each combination of them better results, it is determined that TA interleaving maintains the best performance images for different channel states, being nearly followed by TP interleaving.

\section{ACKNOWLEDGMENT}

This work is supported by Conicyt through Fondecyt Project No. 11121657.

\section{REFERENCES}

[1] I. Akyildiz, W. Su, Y. Sankarasubramaniam, and E. Cayirci, "Wireless sensor networks: A survey," Computer Networks, vol. 38, no. 4, pp. 393-422, March 2002.

[2] T. Arampatzis, J. Lygeros, and S. Manesis, "A survey of applications of wireless sensors and wireless sensor networks," in Proceedings of the 2005 IEEE International Symposium on Intelligent Control, Mediterrean Conference on Control and Automation, June 2005, pp. 719-724.

[3] S. Soro and W. Heinzelman, "A survey of visual sensor networks," Advances in Multimedia, vol. 2009, p. 21 pages, 2009, article ID 640386.

[4] C. Yeo and K. Ramchandran, "Robust distributed multi-view video compression for wireless camera networks," in Visual Communications and Image Processing, vol. 6508, San Jose, CA, USA, January 2007.

[5] C. Duran-Faundez and V. Lecuire, "Error resilient image communication with chaotic pixel interleaving for wireless camera sensors," in REALWSN'08, ACM, Ed., Glasgow, Scotland, UK, April 2008, pp. 21-25.

[6] V. Lecuire, C. Duran-Faundez, and N. Krommenacker, "Energy-efficient transmission of wavelet-based images in wireless sensor networks," EURASIP Journal on Image and Video Processing, vol. 2007, pp. Article ID 47345, 11 pages, 2007, doi:10.1155/2007/47345.

[7] H. Wu and A. A. Abouzeid, "Error resilient image transport in wireless sensor networks," Computer Networks, vol. 50, no. 15, pp. 2873-2887, October 2006.

[8] M. Wu and C. W. Chen, "Multiple bitstream image transmission over wireless sensor networks," in Proceedings of IEEE Sensors, vol. 2, October 2003, pp. 727-731.

[9] M. A. M. M. El-Bendary, A. A. El-Azm, N. El-Fishawy, F. S. M. AlHosarey, M. A. R. Eltokhy, F. E. A. El-Samie, and H. B. Kazemian, "An efficient chaotic interleaver for image transmission over ieee 802.15.4 zigbee network," Journal of Telecommunications and Information Technology, 2011.

[10] C. Duran-Faundez, V. Lecuire, and F. Lepage, "Tiny block-size coding for energy-efficient image compression and communication in wireless camera sensor networks," Signal Processing: Image Communication, vol. 26 , no. 8-9, pp. $466-481,2011$.

[11] C. J. Turner and L. L. Peterson, "Image transfer: an end-to-end design," in Proceedings of the SIGCOMM '92 Symposium. Baltimore, Maryland: ACM Press, 1992, pp. 258-268.

[12] E. J. Posnak, S. P. Gallindo, A. P. Stephens, and H. M. Vin, "Techniques for resilient transmission of jpeg video streams," in In Proceedings of Multimedia Computing and Networking, Septiembre 1994, pp. 243-252.

[13] E. Orellana-Romero, J. SanMartin-Hernandez, C. Duran-Faundez, V. Lecuire, and C. Aguilera, "Sim-lit: A simulation framework for image quality assessment in wireless visual sensor networks under packet loss conditions," in SCCC'2011, 2011.

[14] SIPI, USC, "Sipi image database - volume 3: Miscellaneous," 2013, last Visited: 05/07/2013. [Online]. Available: http://sipi.usc.edu/database/ database.php?volume $=$ misc 